\section{Cronobacter sakazakii clinical isolates overcome host barriers and evade the} immune response

Faisal S. Almajed ${ }^{1,2}$ \& Stephen J. Forsythe ${ }^{1}$

${ }^{1}$ Pathogen Research Group

School of Science and Technology

Nottingham Trent University

Clifton Lane

Nottingham, UK. NG 11 8NS

${ }^{2}$ College of Applied Medical Sciences

King Saud bin Abdulaziz University for Health Sciences

Riyadh, 11426, Saudi Arabia

Corresponding author: Prof SJ Forsythe. Email: Stephen.forsythe@ntu.ac.uk. 


\section{Abstract}

Cronobacter sakazakii is the most frequently clinically isolated species of the Cronobacter genus. However the virulence factors of $C$. sakazakii including their ability to overcome host barriers remains poorly studied. In this study, ten clinical isolates of $C$. sakazakii were assessed for their ability to invade and translocate through human colonic carcinoma epithelial cells (Caco-2) and human brain microvascular endothelial cells (HBMEC). Their ability to avoid phagocytosis in human macrophages U937 and human brain microglial cells was investigated. Additionally, they were tested for serum sensitivity and the presence of the Cronobacter plasminogen activation gene (cpa) gene, which is reported to confer serum resistance.

Our data showed that the clinical C. sakazakii strains invaded and translocated through Caco-2 and HBMEC cell lines and some strains showed significantly higher levels of invasion and translocation. Moreover, C. sakazakii was able to persist and even multiply in phagocytic macrophage and microglial cells. All strains, except one, were able to withstand human serum exposure, the single serum sensitive strain was also the only one which did not encode for the cpa gene. These results demonstrate that $C$. sakazakii clinical isolates are able to overcome host barriers and evade the host immune response indicating their capacity to cause diseases such as necrotizing enterocolitis (NEC) and meningitis. Our data showed for the first time the ability of $C$. sakazakii clinical isolates to survive and multiply within human microglial cells. Additionally, it was shown that $C$. sakazakii clinical strains have the capacity to translocate through the Caco-2 and HBMEC cell lines paracellularly. 


\section{Introduction}

The Cronobacter genus is a member of the Enterobacteriaceae family. It comprises a distinct group of Gram-negative bacilli that are catalase-positive, oxidase-negative, non-spore forming, facultatively anaerobic, and motile via peritrichous flagella [1-3]. The Cronobacter genus contains 7 different species including $C$. condimenti, $C$. dublinensis, C. malonaticus, C. muytjensii, C. sakazakii, C. turicensis, and C. universalis [4-6]. C. sakazakii isolates represent $72.1 \% \quad(n=1400)$ of the total Cronobacter genus isolates in the open access Cronobacter PubMLST database (http://www.pubmlst.org/cronobacter/), and this species has been linked to several fatal NEC and meningitis cases around the world [7-10]. C. sakazakii have been isolated from prepared infant feeds associated with neonatal intensive care unit (NICU) infections. Cases of necrotising enterocolitis (NEC), bacteraemia, and meningitis have a $40-80 \%$ mortality rate, and $20 \%$ of the survivors develop serious neurological disorders [11-14]. C. sakazakii distinct pathovars which are clonal lineages, of particular clinical significance being clonal complex 4 (CC4) that contains sequence type 4 (ST4), as well as ST12. These are strongly associated with invasive meningitis and NEC cases, respectively [15]. One of the most studied NICU outbreaks was in 1994 when 3 infants died from infections by C. sakazakii ST4 strains [14].

For organisms to establish a systemic infection they must adhere to the host cell, translocate to the underlying tissues, and then disseminate throughout the body. Therefore, the intestinal epithelium has an important role in protecting the body against bacterial invasion. Once this layer loses its integrity, the invading organism can infect the tissue beneath [16]. The ability of $C$. sakazakii to invade the intestinal epithelium and brain endothelium is therefore a crucial step for its pathogenesis. It was shown previously that $C$. sakazakii has the ability to adhere to epithelial and endothelial cells in vitro [13, 17]. A study by Townsend et al. [18] used isolates from the French outbreak in 1994, and showed that the C. sakazakii strains were able to adhere and invade Caco- 2 and rat brain capillary endothelial cells (rBCEC4) cell lines. Moreover, the organism was able to persist and multiply within the human macrophage U937 cell line [19]. Another study by Giri et al. [12] showed that food and environmental strains of $C$. sakazakii have the ability to invade the HeLa subline 
INT407 (human embryonic intestinal cells) and human brain microvascular endothelial cells (HBMEC).

The translocation process of the organism follows the initial attachment and invasion phases. It is the step that initiates the pathogenesis at the next tissue level after passing through the epithelial layer. Townsend et al. [20] reported that the presence of lipopolysaccharide (LPS) in infant formula increased the permeability of the intestinal epithelium leading to the translocation of $C$. sakazakii. Giri et al. [12] showed that the invasive food and environmental $C$. sakazakii strains were able to translocate intracellularly through the intact monolayers of the Caco-2 and HBMEC cell lines. This suggests that the bacterium is able to overcome the physical host barriers in intestines and CNS.

A number of virulence traits have been identified in Cronobacter, which may facilitate the invasion and dissemination of the organism in the host. Franco et al. [21] reported that the plasmid-borne Cronobacter plasminogen activator (Cpa) may provide resistance to bactericidal activity of serum through cleaving complement components $\mathrm{C} 3$ and $\mathrm{C} 4 \mathrm{~b}$, and the activation of plasminogen and inactivation of $\alpha 2$ AP. In a study of over 100 Cronobacter genomes, cpa was found in C. sakazakii and not C. malonaticus [23; http://pubmlst.org/cronobacter/], and therefore may contribute to the higher clinical incidence of this species. It has also been reported that the outer membrane protein $\mathrm{A}(\mathrm{OmpA})$ of Cronobacter spp. has a role in the colonisation of the gastrointestinal tract (GIT) [21, 23]. Also, it was demonstrated that the outer membrane proteins OmpA and OmpX were required for the basolateral invasion of enterocyte-like human epithelial cells by C. sakazakii [23]. Singamsetty et al. [24] demonstrated that the entry of Cronobacter spp. into HBMEC requires ompA expression and depends on microtubule condensation in these cells. This might help in the invasion of human intestinal cells and invasion of the brain endothelial cells to cause meningitis [25]. Moreover, it was recently shown that $C$. sakazakii ST4 strain 767 was able to produce outer membrane vesicles (OMVs) that have the capacity to increase the host's cell prolifration and stimulate a pro-inflammatory innate immune response [26].

This study used clinical isolates of $C$. sakazakii which had been previously genotyped by multilocus sequence typing (MLST), and many of which had been whole genome sequenced [23; http://pubMLST.org/cronobacter/]. The research aim was to study the virulence potential and pathogenicity of well characterised $C$. 
144 sakazakii clinical isolates and their ability to overcome host physical barriers and

145 evade host immune response.

\section{Results}

148

149 2.1. Invasion efficiencies of $C$. sakazakii clinical isolates to Caco-2 and HBMEC cell

150 lines

151

152 The invasion assay, using gentamicin protection to kill the extracellular bacteria, was

153 used to assess the ability of 10 C. sakazakii clinical isolates to invade the Caco-2

154 and HBMEC cell lines. With regard to the Caco-2 cell line, different invasion levels

155 were noted among these isolates, and strain 695 was the most significant $(P<0.05)$.

156 The level of invasion by 695 was as high as $S$. Enteritidis, which was used as

157 positive control strain for the assay. Strains $20,767,1221$, and 696 were moderate in

158 invasion, whereas strains 1240, 1242, 1249, 658 and 680 were low (Fig.1).

159 Regarding the HBMEC cell line invasion, strain 767 was the most significant $(P<0.01)$

160 being as high as Cit. koseri, the positive control. The other strains were moderate

161 except for strains 658 and 680, which were the lowest (Fig.1). 


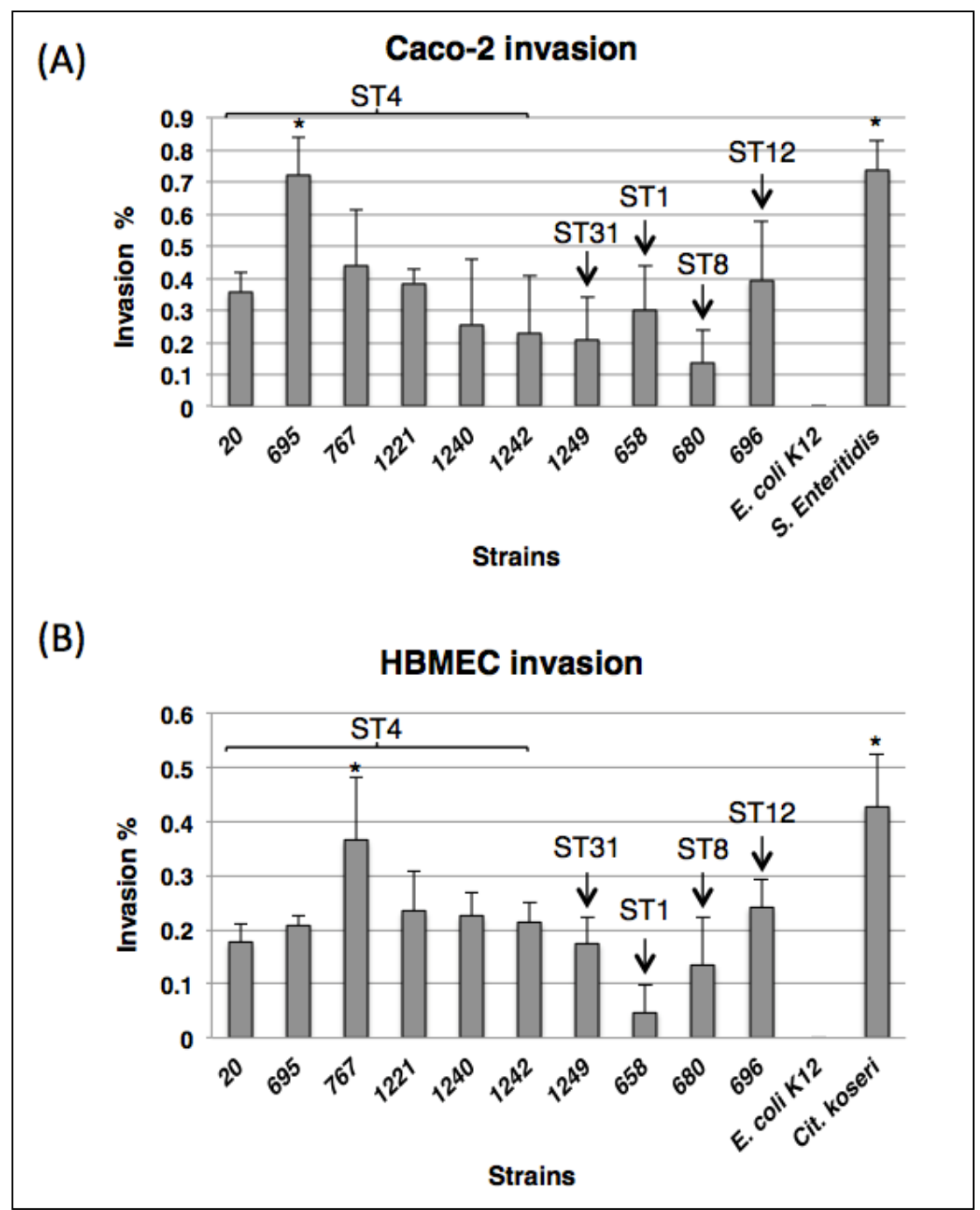

Fig.1. C. sakazakii invasion assay using Caco-2 (A) and HBMEC (B) cell lines over 3 167 hours of incubation showing the differences in invasion levels among strains. The 168 displayed data are the mean \pm standard deviation of invasion efficiency \% of the initial 169 inoculum $\left(10^{6} \mathrm{cfu} / \mathrm{ml}\right)$ of two independent experiments in triplicate. The asterisks 170 above the bars indicate statistically significant differences ( ${ }^{*} P<0.05$; Kruskal-Wallis). 
176 monolayers

178 The aforementioned results showed that $C$. sakazakii isolates were able to invade 179 Caco-2 and HBMEC monolayers. Therefore, these isolates were tested for their 180 ability to translocate through the polarised monolayers of the Caco-2 and HBMEC 181 cell lines. C. sakazakii strain 695 was the highest in translocating through the Caco-2 182 cell line over 5 hours of infection $(P<0.01)$. The other strains including 767,1221 , $1831240,1242,1249,658$, and 696 were moderate, while strains 20 and 680 were the 184 lowest (Fig.2). With regard to the HBMEC cell line, strains 20,695, 1221, 1240, and 185696 were high in translocation $(P<0.01)$, and strain 767 was the most significant over 1865 hours of incubation $(P<0.001)$. C. sakazakii strains 1242 and 1249 were moderate, 187 whereas 658 and 680 were the lowest (Fig.2). It was noted that the transepithelial 188 electrical resistance (TEER) declined over the period of the experiment. The higher 189 drops of TEER were accompanied with high translocation levels (Fig.3), suggesting 190 that $C$. sakazakii clinical isolates might translocate through the Caco-2 and HBMEC 191 cell lines paracellularly.

192 


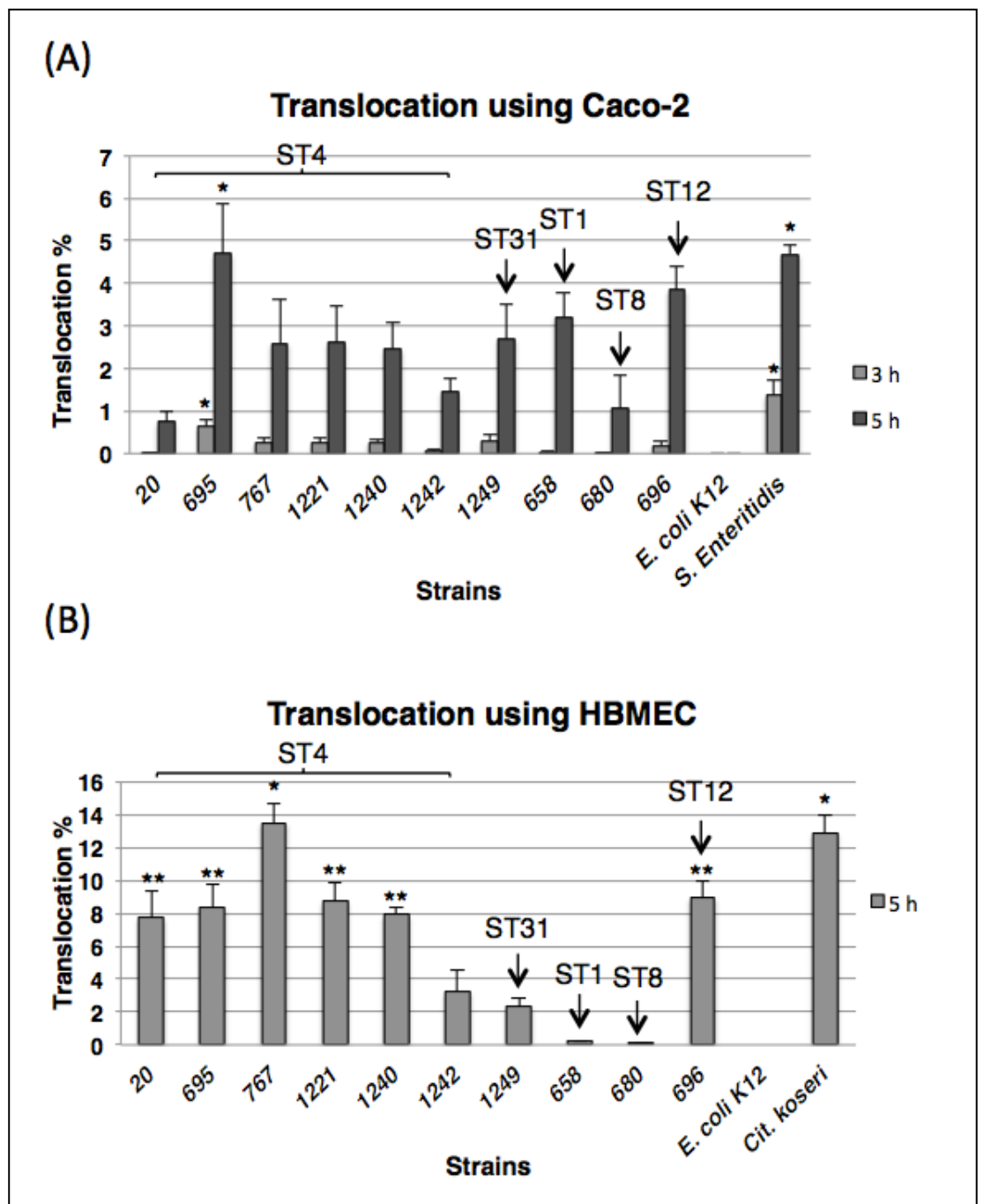

Fig.2. C. sakazakii translocation assay using Caco-2 (A) and HBMEC (B) cell lines over 5 hours of incubation showing the differences in translocation ability among strains. The displayed data are the mean \pm standard deviation of translocation efficiency $\%$ of the initial inoculum $\left(10^{6} \mathrm{cfu} / \mathrm{ml}\right)$ of two independent experiments. The asterisks above the bars indicate statistically significant differences $\left({ }^{*} P<0.001\right.$, ** $P<0.01$; Kruskal-Wallis). 


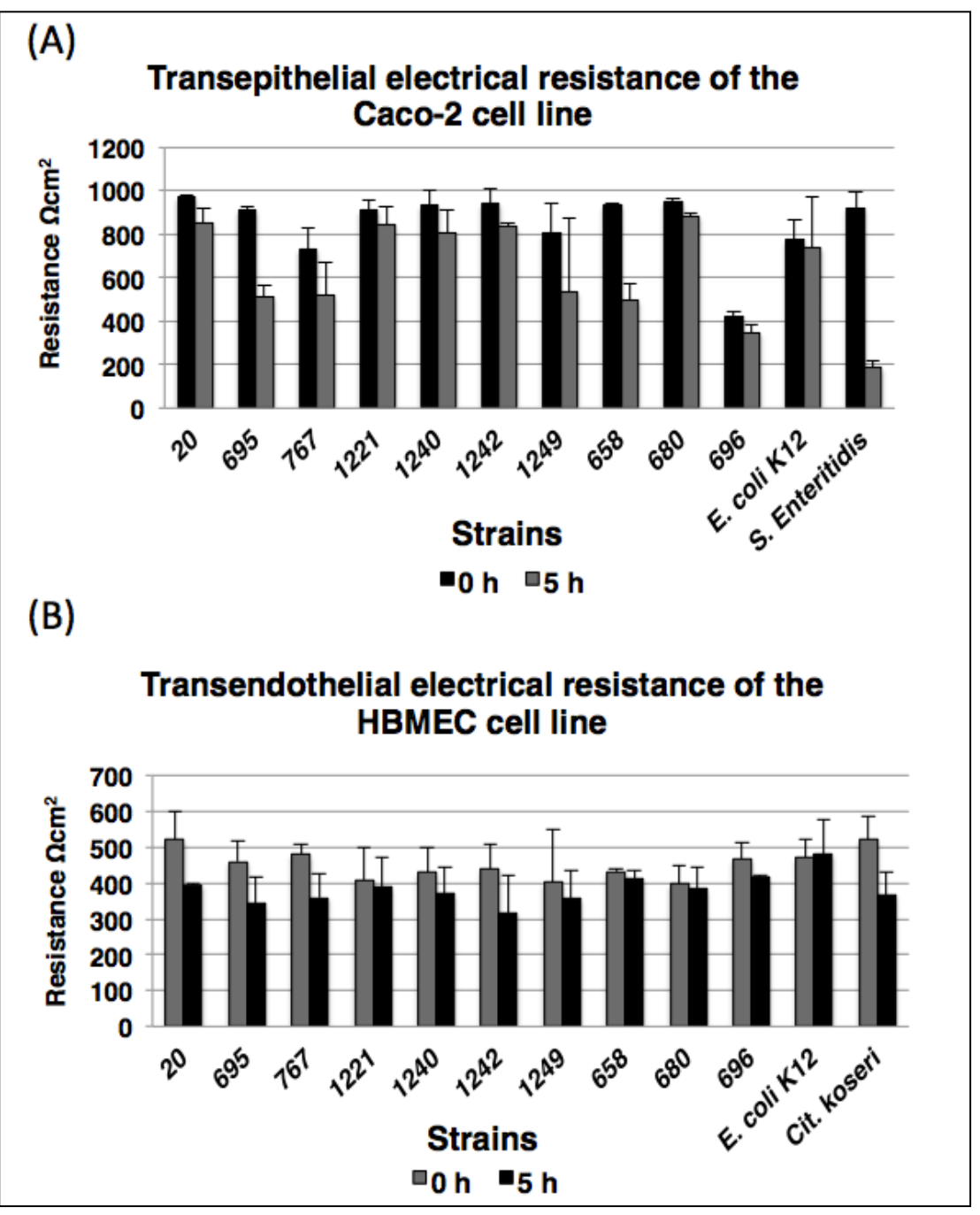
transendothelial electrical resistance (TEER) of the HBMEC cell line (B) over 5 hours of incubation, showing changes in resistance over time. 
C. sakazakii clinical strains in this study $(n=10)$ were tested for their cytotoxic impact using the MTT assay. Apart from strain 680 , all the other strains were able to induce cell death of the Caco- 2 cell line, as the assay displayed declined absorbance levels after 3 hours of incubation indicating low MTT reduction. Although strains 1221 , 1240 , and 1249 did not follow the same pattern, they however showed lower absorbance when compared to strain 680 . This suggests that these strains, to some extent, are able to induce cell death more than strain 680 (Fig.4). With regard to the HBMEC cell line, it did not show susceptibility to cytotoxicity over the first hour of the assay in contrast to Caco-2 cells; nevertheless after prolonged incubation for 3 hours the cytotoxic effect appeared to be increased (Fig.4). 


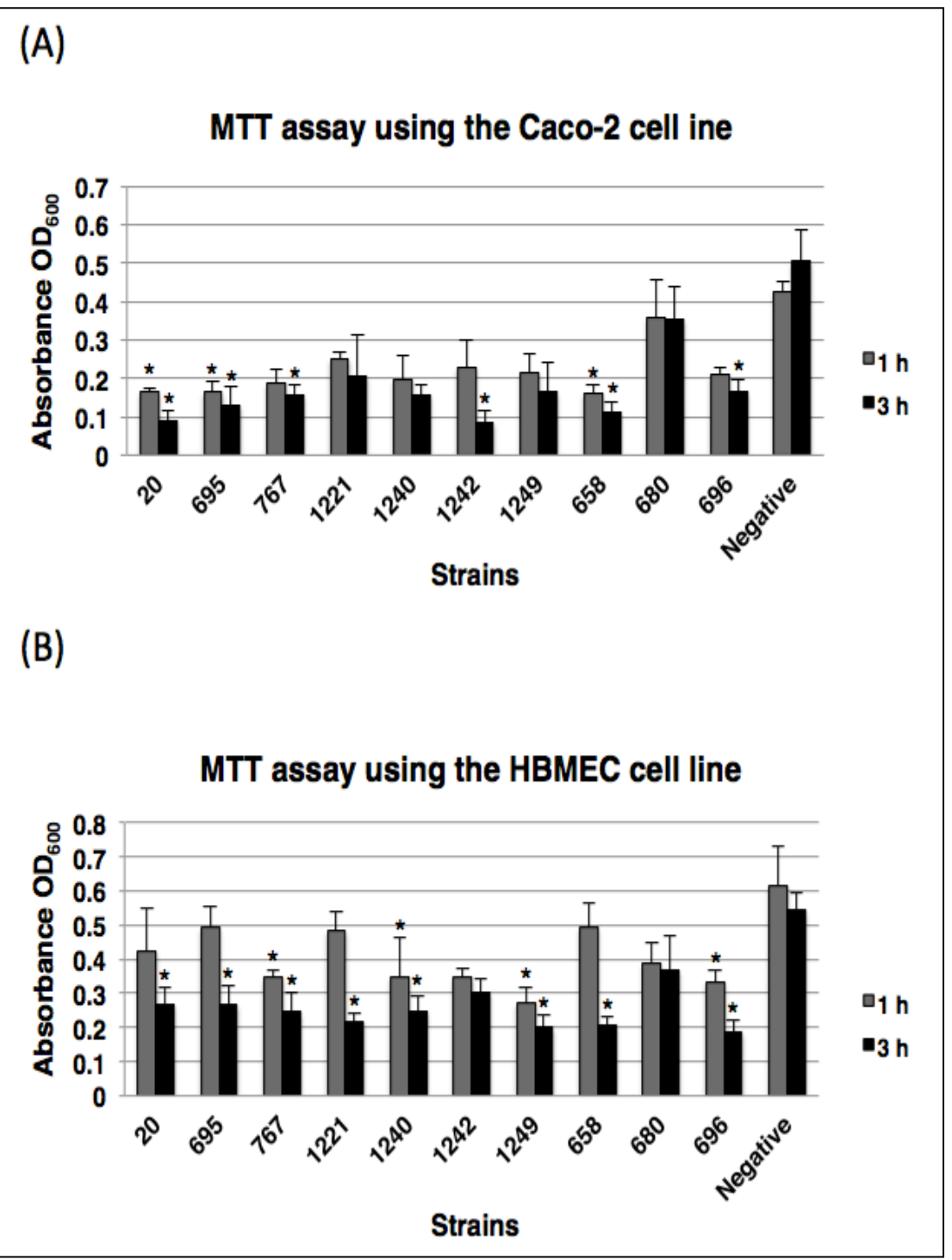

Fig.4. Cytotoxicity of C. sakazakii strains on Caco-2 (A) and HBMEC (B) up to 3 hours of incubation. MTT reduction was used to measure the cytotoxicity levels of $C$. sakazakii strains where only the viable HBMEC cells are able to reduce MTT to its insoluble purple form formazan, the higher absorbance $\left(\mathrm{OD}_{600}\right)$ the higher in MTT reduction (low toxicity) and vice versa. The negative control used was uninfected cells treated using the same protocol with no bacteria added. The data presented in mean \pm standard error of mean of three independent experiments. The asterisks above the bars indicate statistically significant differences between the strains in this experiment $\left({ }^{*} P<0.05\right.$; ANOVA). 
The survival within macrophages is an important indicator of the pathogenicity of the organism. It enables the persistent bacterium to evade the immune response inside the host. C. sakazakii clinical isolates were tested for their ability to survive within human macrophages using $U 937$ cell line. All strains were taken up by these cells and showed persistence for up to 72 hours (Fig.5). However, strain 680 declined significantly after 72 hours. The other strains demonstrated different levels of multiplication at 24 hours and strains $20,695,767,1221,658$, and 696 were the most significant $(P<0.05)$.

\subsection{C. sakazakii survival within human microglial cells}

Microglial cells are brain resident macrophages, which respond rapidly to the presence of the pathogens and brain damage. Furthermore, they perform phagocytosis, antigen presentation, and are responsible for cytokine secretion. Microglial cells are able to migrate to the injured brain tissues to remove the damaged ones [27]. C. sakazakii is linked to fatal meningitis cases, and therefore it is important to consider its ability to resist phagocytosis and withstand killing inside the brain. This experiment was conducted to assess the ability of $C$. sakazakii to survive within microglial cells and multiply intracellularly. C. sakazakii clinical strains were able to survive up to 72 hours post infection (Fig.5). However, strains 695, 1242, 658, and 696 showed lower survival levels. Additionally, strain 680 was taken up and killed rapidly following the uptake. Strains $20,767,1221,1240$, and 1249 multiplied significantly at 24 hours $(P<0.001)$. 


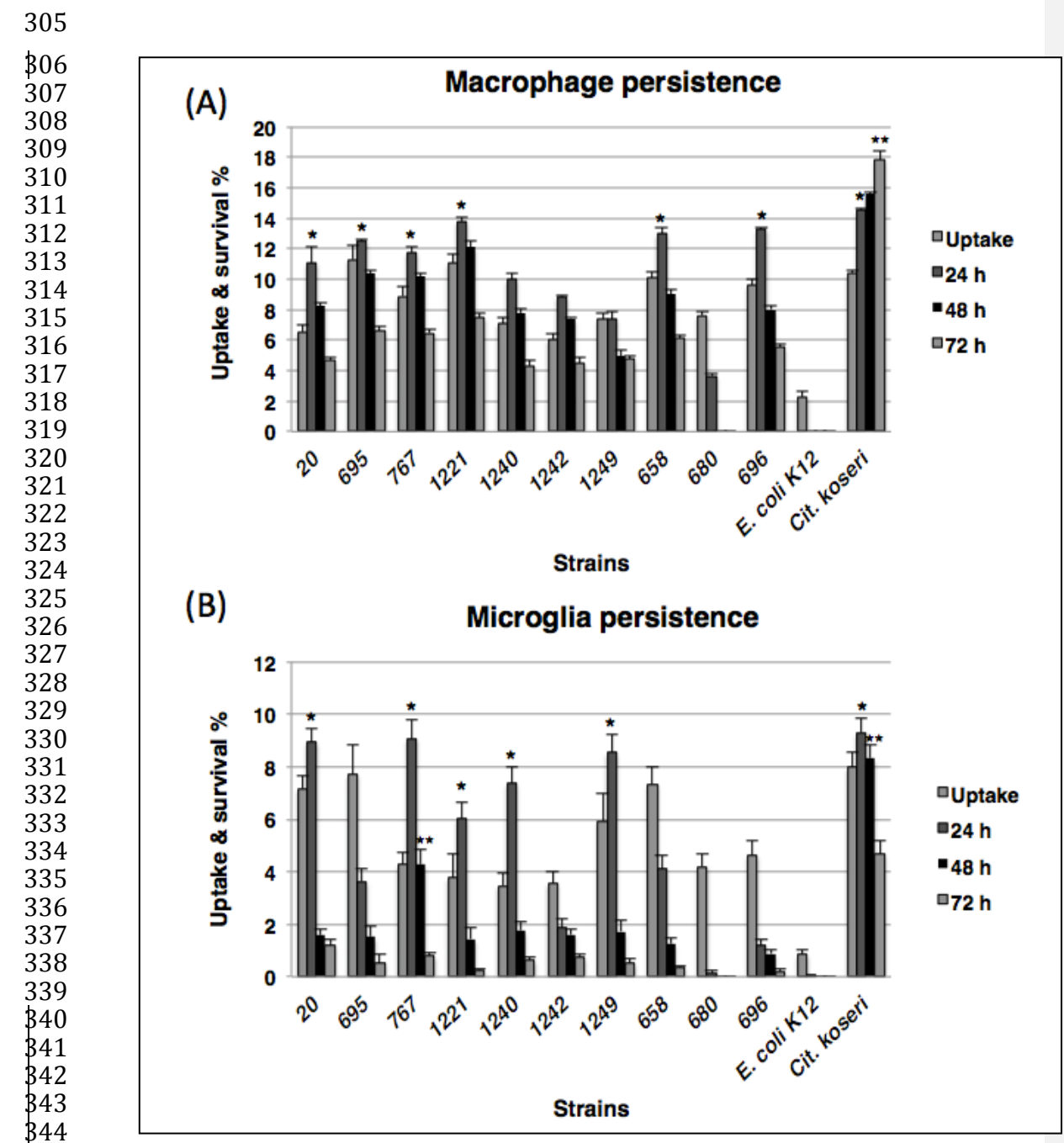




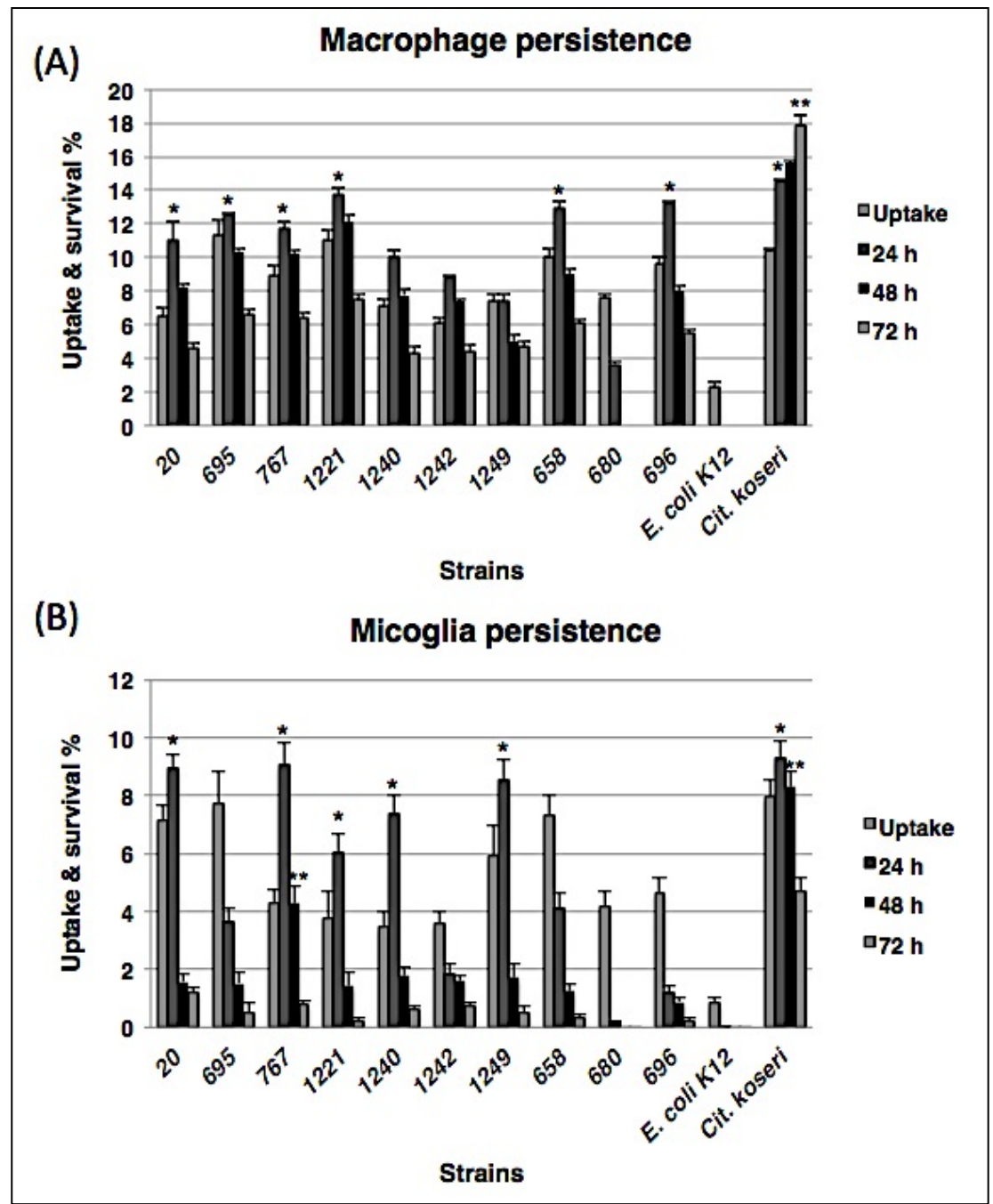

Fig.5. C. sakazakii uptake and persistence assay using human macrophage (A) and human microglia (B) cell lines over 72 hours of incubation showing the differences in survival among strains. The displayed data are the mean \pm SEM for uptake and persistence efficiency $\%$ of the initial inoculum $\left(10^{5} \mathrm{cfu} / \mathrm{ml}\right)$ of three independent experiments. The asterisks above the bars indicate statistically significant differences $\left({ }^{*} P<0.05,{ }^{* *} P<0.001\right.$; ANOVA). 
All C. sakazakii strains in this experiment $(n=10)$ showed up to $60 \%$ decrease in their viable count at the first hour incubation with human serum (Fig.6). After the second hour of incubation, it was noted that $9 / 10$ strains showed an increase in their viable numbers, whereas strain 680 showed a $30 \%$ reduction in its viability. The majority of the strains were able to survive in human serum and increased by up to 4 -fold in their numbers after 3 hours of incubation. The exception was strain 680 , which was serum sensitive and its growth declined dramatically to $60 \%(P<0.05)$. Strains 20,1242 , 1249 , and 696 were the highest in serum tolerance showing considerable elevated growth rates $(>200 \%)$ during the period of the assay.

Franco et al. [21] reported that the outer membrane protease Cpa of Cronobacter is responsible for serum resistance. All C. sakazakii clinical strains in this research were tested for the presence of the cpa gene using PCR probing and BLAST search. Most $(9 / 10)$ of the strains were confirmed positive for this gene. These strains demonstrated resistance to human serum except for strain 680 , which lacked the presence of cpa gene in PCR and BLAST search and was serum sensitive. It was shown that strain 680 lack the presence of pESA3 plasmid, but on the other hand a pESA3-like plasmid was present in this strain, which lacked some plasmid-borne virulence genes [22]. 


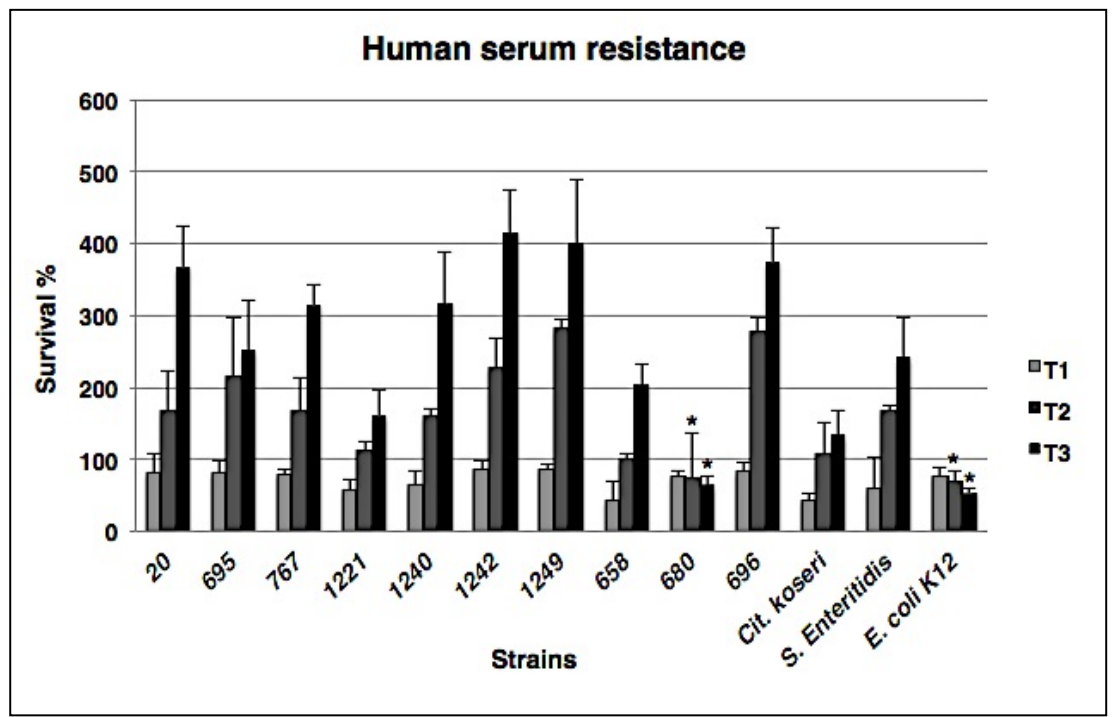

Fig.6. Sensitivity of $C$. sakazakii to human serum over 3 hours of incubation showing the difference in growth among strains overtime. Most of the strains showed increases in their viable counts, and strains 6 (ST4), 680 (ST4), and E. coli K12 showed significantly declined values. The displayed data are the mean \pm standard deviation of survival \% $\left(10^{6} \mathrm{cfu} / \mathrm{ml}\right.$ initial inoculum) of two independent experiments. The asterisks above the bars indicate statistically significant differences $\left({ }^{*} P<0.05\right.$; Kruskal-Wallis).

\section{Discussion}

This study used a range of clinical isolates of $C$. sakazakii. These strains were diverse with respect to their temporal and geographical sources, and all but one strain were from patients with clinical presentations. The exception was $C$. sakazakii 658 (ATCC BAA-894) which had been isolated from the formula, that was not intended for infants, used in the fatal NICU C. sakazakii outbreak at the University of Tennessee [7]. The corresponding CSF isolate has not been deposited in any international culture collection and therefore the PFGE indistinguishable strain (ATCC code BAA-894) was used instead. This was also the first genome-sequenced strain of Cronobacter, including the pESA3 plasmid which encodes for the cpa gene [2]. 
Tissue culture assays were applied to examine the bacterial-host interaction and to assess the ability of $C$. sakazakii strains to overcome human intestinal and brain barriers represented by the Caco-2 and HBMEC cell lines. All the strains were able to invade the Caco-2 cell line (Fig.1). However, there was strain to strain variation in the level of invasion, with strain 695 (ST4) being the most invasive and was similar to that of the positive control $S$. Enteritidis strain $(P<0.05)$. With reference to HBMEC cell line invasion, most of the strains showed moderate invasion levels and strain 767 (ST4) displayed significant high invasion level $(P<0.01)$. Whereas, strains 658 (ST1) and 680 (ST8) showed the lowest invasion (Fig.1).

Overall, the invasion results indicated that the majority (9/10) of the strains were able to invade the cell lines and therefore potentially translocate towards the underlying tissues and blood stream. Consequently, this could lead to the dissemination of the organism around the body. To further investigate the translocation process further, assays using the same human cell lines were applied. All strains were able to translocate through the Caco-2 cell line especially strain 695 that displayed significant high translocation ability $(P<0.01)$, while strain 696 (ST12) was moderate (Fig.2). These strains were able to cause high cytotoxicity levels to the Caco-2 cell line, which could lead to increase the cell line permeability causing more bacterial translocation (Fig.4). Strain 695 (ST4) accounted for a fatal neonatal NEC infection while strain 696 (ST12) was previously linked to neonatal NECII infection [14]. The invasion and translocation results correlate their virulence and their ability to cause that disease.

There was a significant decrease in TEER when using the invasive and translocating strains indicating a disruption in the tight junctions (Fig.3). This might explain the mechanism of translocation for the strains where they can migrate in between the cells by altering the tight junctions. This might trigger the onset of NEC, which could lead to the spread of the bacteria in the blood stream. This clinical presentation of the organism indicates the potential to reach the brain microvascular endothelium and invades its cells. Therefore the translocation assay using the HBMEC cell line was initiated to investigate whether the organism has the ability to translocate through these cells or not. ST4 strains 20,695, 1221, and 1240 in addition to 696 (ST12) were high in translocation $(P<0.01)$, and strain 767 (ST4) was the most significant in translocation over 5 hours of incubation ( $P<0.001$; Fig.2). C. sakazakii strains 1242 (ST4) and 1249 (ST31) were moderate in translocation, whereas 658 (ST1) and 680 (ST8) were the lowest. Strains 695 (ST4), 767 (ST4), and 696 (ST12), which showed 
high translocation rates were the highly cytotoxic strains to the HBMEC cell line (Fig.4). Strain 767 (ST4) that exhibited the most significant translocation through HBMEC cell line was associated with a fatal meningitis case [14] and also produces cytopathogenic OMVs [26]. Overall, the HBMEC TEER was stable during the first 4 hours of incubation, indicating the stability of the tight junctions and the integrity of the cell line. However, after 5 hours of infection it displayed declined levels with the translocated strains (Fig.3). The decrease in TEER might be attributed to the deterioration of endothelial integrity that could be accredited to the cytotoxic killing to the HBMEC cell line that was caused by those strains.

The translocated strains might need a longer infection period to accomplish the translocation process, as the translocation was noted 5 hours post infection, while in Caco-2 it was after 1 hour with some strains. Moreover, they could use different mechanisms of invasion to overcome this barrier that is a part of the BBB. One of the possible mechanisms that might assist the strains to translocate is the bacterial cytotoxicity that leads to initiate cell death in the cell line via apoptosis or necrosis. Moreover, the cytokines released by the cells might play a role in the same process by making the cell line permeable. High levels of nitric oxide (NO) are a potential factor that could contribute in the permeability of the cell line. Also, OMVs might contribute in this process by triggering the host proinflammatory response leading to the secretion of some inflammatory mediators, including cytokines and NO, that in turn could cause cell line permeability [26]. The translocation of these strains indicated their potential to pass through towards the brain tissues triggering the host response, which could result in brain inflammation and tissue damage.

In a previously published study by Giri et al. [12], non-clinical C. sakazakii isolates were examined and showed their ability to invade and translocate through the Caco2 and HBMEC cell lines intracellularly. In this research, the same cell lines were used to compare the results of the clinical strains in our study and the non-clinical strains from the previous research by Giri et al. [12] with regard to the capacity and mechanism of translocation. However, the resultswe found that of the capacity of translocationtranslocation ability of $C$. sakazakii clinical isolates that were obtained by our research were-was more than 10 times higher than the previous study using the same cell lines. Additionally, our results showed-suggested a different mechanism of translocation, which istheir capacity of paracellular translocation through those cell lines. All of the strains were from clinical sources and were linked to severe and fatal neonatal cases. 
493 The survival studies were conducted to investigate the ability of the test strains to 494 survive and multiply within macrophages and microglia which are both types of 495 immune cells. C. sakazakii strains used in the survival experiments showed the 496 ability to persist within human macrophages cell line U937 for up to 72 hours of 497 incubation. Moreover, ST4 strains 20, 767, and 1221 in addition to 658 (ST1) were 498 able to survive and multiply significantly $(P<0.001)$. The survival results were 499 comparable to the results obtained previously by Townsend et al. [18]. Strains 767 500 (ST4) and 696 (ST12) showed similar persistence and multiplication levels as the $\$ 01$ ones published in that research. However, Townsend et al. [18]_reported that strain 502695 (ST4) was able to survive and could not multiply within macrophages. These 503 results are in contrast of the ones obtained here as strain 695 (ST4) showed the 504 ability to survive and multiply in U937 cells. Our results correlate the virulence 505 potential of the strain, and the invasion and translocation profiles, as it was an 506 invasive strain to both of the Caco-2 and HBMEC cell lines. Moreover, it is a clinical 507 strain that was linked to a fatal NEC infection [14]. This suggests that this strain was 508 able to establish a successful infection and has the virulence traits to avoid 509 phagocytic killing.

The survival and multiplication within macrophages could help the organism to use macrophages as a vehicle to invade the other body organs. This mechanism is called the "Trojan horse" and where the organism translocates through tissues inside macrophages. This mechanism allows the bacterium to hide inside the phagocytic cells, escape from the immune response, and reach the other body organs such as the brain [28]. Some cytokines secreted by the infected tissues, such as IL-8, attract phagocytic cells and make these tissues permeable and leaky allowing the immune cells to migrate to the site of infection, and help in increasing the number of the invading organism [29-31]. The damage could be indicated by the host response induced by the bacterium. Moreover, the persistent strains were confirmed to be serum resistant, and these two characteristics enhanced their ability to avoid the host immune response and cause bacteraemia, which could be advantageous for the organism to migrate through the BBB endothelium.

Microglia are brain resident innate immune cells that are responsible for phagocytosis as well as the ability to produce inflammatory mediators such as NO and TNF- $\alpha[27,32,33]$. Although their ability to eliminate $C$. sakazakii CNS infection in vitro has not yet been examined, we are the first to report the ability of $C$. sakazakii 
to survive within human microglial cells. It was shown in this research that the majority of $C$. sakazakii strains were able to persist in human microglia as represented by the HMGC cell line for 72 hours. Moreover, ST4 strains 20, 767, 1221 , and 1240 in addition to 1249 multiplied significantly in this cell line $(P<0.001)$. Although these strains showed the ability to multiply within microglia, they nevertheless showed declined levels of persistence afterwards. The ability of the bacteria to reproduce intracellularly within these phagocytic cells demonstrates their virulence potential to withstand the bactericidal activity of microglia and evade the host immune response.

Invasive microorganisms have protective mechanisms against serum-mediated killing. Bacterial structures including outer membrane proteins and proteases were identified for their roles to avoid this bactericidal action [34-36]. Franco et al. [21] showed that the $\mathrm{Cpa}$ is a plasminogen activator that plays an essential role in Cronobacter serum resistance. Nine of the ten $C$. sakazakii strains in this research were regarded as serum resistant being able to replicate in human serum and appeared to be completely refractory to serum killing. Whereas $C$. sakazakii strain 680 was serum sensitive and showed significant reduction in viability $(P<0.05$; Fig 6$)$.

Franco et al. [21] further showed that a cpa mutant of C. sakazakii BAA-894 (synonym for 658 in this study) was serum sensitive compared with the wild type. In our study $9 / 10$ C. sakazakii strains were serum resistant and also encoded for the cpa gene. The exception being strain 680 which lacked this gene and was serum sensitive. While strain 680 was the only ST8 strain studied here, a BLAST search of the PubMLST database revealed the cpa gene is absent from all $(n=8)$ genome sequenced ST8 strains. This absence could explain the observation by Forsythe et al. [15] in a review of $>1000$ Cronobacter strains, that C. sakazakii ST8 is not associated with severe Cronobacter infections [8]. By inference therefore, cpa could be an important factor in $C$. sakazakii resistance to serum killing in the host and enabling dissemination around the neonate's body.

In conclusion, C. sakazakii clinical isolates showed the ability to invade and translocate through Caco-2 and HBMEC cell lines. Moreover, they demonstrated the ability to persist and multiply within macrophages and microglial cells. Additionally, the isolates were resistant to human serum bactericidal effect. However, it was noted that strain 680 did not follow the same pattern of virulence and pathogenicity as it was low in invasion, translocation, and phagocytosis survival in addition to being 
sensitive for human serum killing. This might be attributed to lack of the pESA3 plasmid [22], and hence other plasmid-borne virulence genes such as cpa, and other genes involved in invasion, translocation, and phagocytosis survival. Although this strain was a CSF isolate it demonstrated a weakness in pathogenicity to HBMEC and microglial cells in vitro. It was reported that $C$. sakazakii ST12 has been associated with cases of necrotizing enterocolitis [15]. It was shown in this study that strain 696 (ST12) was able to invade and translocate through the Caco-2 cell line in addition to its ability to induce cytotoxicity to the same cell line. This might indicate its ability to induce necrotizing enterocolitis in vivo. Furthermore, it was observed that the most invasive and translocated strains in this research were in the $C$. sakazakii ST4 clonal complex, which is the lineage that linked to the most neonatal meningitis cases worldwide [37]; Table 1. ST4 strains such as 695, 767, 1221, 1240, and 1242 within clonal complex 4 were invasive and highly translocated isolates and were responsible for fatal NEC and meningitis infections. This indicates their ability to translocate through the gut mucosa and BBB in vivo and cause NEC and meningitis. It is important to further study the strains among this clonal complex and discover their pathogenicity traits and their role in triggering the host response and its outcome.

\section{Materials and methods}

\subsection{Bacterial strains}

Ten C. sakazakii strains were used in this research (Table.1). These isolates were from Nottingham Trent University culture collection. Additional metadata for all strains can be obtained from the open access Cronobacter PubMLST database: www.pubmlst.org/cronobacter/. The strains were chosen according to their source and clinical outcomes, and well-characterised strains from the 1994 French outbreak and University of Tennessee [7, 14]. For routine culturing the strains were grown on TSA (Oxoid, UK) under aerobic conditions at $37^{\circ} \mathrm{C}$ for overnight. 
Table.1

Cronobacter sakazakii strains used in this study

608

\begin{tabular}{ccccc}
\hline Strain & $\begin{array}{c}\text { Sequence } \\
\text { type (clonal } \\
\text { complex) }\end{array}$ & Source & $\begin{array}{c}\text { Clinical } \\
\text { presentation }\end{array}$ & Country \\
\hline 20 & 4 (CC4) & Clinical & Unknown & Czech Republic \\
695 & 4 (CC4) & Clinical - Trachea & Fatal NEC II & France \\
767 & 4 (CC4) & Clinical - Trachea & Fatal meningitis & France \\
1221 & 4 (CC4) & Clinical - CSF & Meningitis & USA \\
1240 & 4 (CC4) & Clinical - CSF & Fatal meningitis & USA \\
1242 & $4($ CC4) & Clinical - Brain & Fatal meningitis & USA \\
1249 & 31 (CC31) & Clinical & Fatal infant isolate & UK \\
$658^{a}$ & 1 (CC1) & NIFNIF & Meningitis & USA \\
680 & 8 (CC8) & Clinical - CSF & Unknown & USA \\
696 & 12 & Clinical - Faeces & NEC II & France \\
\hline
\end{tabular}

609 NEC: Necrotising enterocolitis. CSF: Cerebrospinal fluid. CC: clonal complex. NIF: Non-infant formula.

610 a Also known as C. sakazakii BAA-894.

Human colonic carcinoma epithelial cells (Caco-2) passages 17 to 45 acquired from the European Collection of Cell Cultures (ECACC \#86010202) and human brain microvascular endothelial cells passages 2 to 25 (HBMEC; ref. \#HMG030 Inooprot,

617 Spain). Macrophage cell line (U937) passage 12 was obtained from American Type 618 Culture Collection (ATCC; \#CRL-1593.2), and human microglial cell line passage 3 619 was obtained from Innoprot Technologies (Ref.\# P10354). All experiments were 620 applied at consistent conditions of time, temperature, cell line passage, mammalian 621 cells concentration, and bacterial suspension. Salmonella Enteritidis strain NTU 358 622 was used as positive control for Caco-2 cell line, while Citrobacter koseri strain NTU 62348 was the positive control for HBMEC, macrophages, and microglial cell lines. Escherichia coli K12 strain NTU 1230 was the negative control for all cell lines.

\subsection{Bacterial invasion of mammalian cells}

628 This experiment was as described previously by Townsend et al. [18] with slight modifications. Caco-2 cells were grown in Minimum Essential Medium (MEM)

630 supplied with $10 \%(\mathrm{v} / \mathrm{v})$ foetal calf serum (FCS), $1 \%(\mathrm{v} / \mathrm{v})$ non-essential amino acid 631 (NEAA), and 1\% (v:v) penicillin-streptomycin (Sigma Aldrich, UK). HBMEC were 
grown in Dulbecco's modified eagle medium (DMEM) with 10\% (v/v) FCS and 1\% (v/v) penicillin-streptomycin (Sigma Aldrich, UK). Mammalian cells then were seeded at $4 \times 10^{4}$ cell/well in growth medium for 48 hours in $5 \% \mathrm{CO}_{2}$ at $37^{\circ} \mathrm{C}$ to achieve a confluent monolayer. C. sakazakii strains were grown in LB broth for overnight at $37^{\circ} \mathrm{C}$. The suspension then was added to the wells at $\mathrm{MOI} 100$, and incubated in $5 \%$ $\mathrm{CO}_{2}$ at $37^{\circ} \mathrm{C}$ for 2 hours. The wells were then washed using PBS (Sigma Aldrich, UK). Then, $0.5 \mathrm{ml}$ of infection medium supplied with $125 \mu \mathrm{g} / \mathrm{ml}(\mathrm{v} / \mathrm{v})$ of gentamicin (Sigma Aldrich, UK) was added and incubated in $5 \% \mathrm{CO}_{2}$ at $37^{\circ} \mathrm{C}$ for 1 additional hour. The wells were then washed with PBS (Sigma Aldrich, UK) before lysing by $1 \%$ (v/v) Triton X-100 (Fisher Scientific, UK), and plated on TSA at $37^{\circ} \mathrm{C}$ for overnight incubation after serial dilution to obtain viable count. Data are presented as the percentage efficiency of invasion.

\subsection{Translocation assay using Caco-2 cell line}

Translocation assay was performed as previously described [12, 38]. However, 0.8 $\mathrm{ml}$ of growth medium was added to the basolateral chamber of Millicell-24 cell culture plate (Millipore, UK). Caco-2 cells, at a concentration of $4 \times 10^{4}$ cell/well in $0.4 \mathrm{ml} /$ well of Minimum Essential Medium (MEM) supplied with $10 \%(\mathrm{v} / \mathrm{v})$ foetal calf serum (FCS), $1 \%(\mathrm{v} / \mathrm{v})$ non-essential amino acid (NEAA), and $1 \%(\mathrm{v} / \mathrm{v})$ penicillinstreptomycin (Sigma Aldrich, UK), were seeded onto a $3 \mu \mathrm{m}$ pore polycarbonate transwell membrane in the apical chamber of the tissue culture plate and incubated in $5 \% \mathrm{CO}_{2}$ at $37^{\circ} \mathrm{C}$. The medium in the apical and the basolateral chambers was changed every 3 days. Millicell ERS-2 Volt-Ohm Meter (Millipore, UK) was used to measure the TEER. The Caco-2 cell line required up to 21 days to form intact polarised monolayers with TEER $300-850 \Omega \mathrm{cm}^{-2}[12,38,39]$. On the day of the assay, the medium in the basolateral chamber was replaced with infection medium. The medium in the apical chamber was removed, and the membrane was washed using $0.4 \mathrm{ml}$ of PBS (Sigma Aldrich, UK). Bacterial suspensions was prepared was added at $\mathrm{MOI} 100$ to the apical chamber. At each time point of incubation, the basolateral chamber was sampled for viable count after serial dilution and inoculation on TSA. The TEER was measured at each time point. Data are presented as the percentage efficiency of translocation.

\subsection{Translocation assay using HBMEC cell line}


This assay was carried out using the protocol described previously [12, 40, 41]. The basolateral chambers of the 24-well plate were filled with $0.510 \mathrm{ml} /$ well of Dulbecco's modified eagle medium (DMEM) with 10\% (v/v) FCS and $1 \%(\mathrm{v} / \mathrm{v})$ penicillinstreptomycin (Sigma Aldrich, UK). Cells, with a concentration of $4 \times 10^{4}$ cell/well in $0.375 \mathrm{ml} /$ well of previous medium, were seeded onto the apical part of collagencoated polytetrafluoroethylene (PTFE) membrane with a pore size of $0.4 \mu \mathrm{m}$ (Transwell-COL; Corning, USA), and incubated in $5 \% \mathrm{CO}_{2}$ at $37^{\circ} \mathrm{C}$. The medium in the apical chamber was changed every 3 days. The transendothelial electrical resistance (TEER) was measured using Millicell ERS-2 Volt-Ohm Meter (Millipore, UK). According to electrical resistance measurements, HBMEC cell line required 5 to 8 days to form intact polarised monolayers with TEER $300-600 \Omega \mathrm{cm}^{-2}[12,42]$. Prior to infecting the cell line, the filter was washed by $0.375 \mathrm{ml} /$ well PBS (Sigma Aldrich, UK), and the medium in the basolateral part was replaced by infection medium. The cell line was infected by $0.375 \mathrm{ml}$ per well bacterial suspension with MOI of 100 . The basolateral chamber was sampled and serially diluted and then plated for viable count on TSA at $37^{\circ} \mathrm{C}$ before replacing with a fresh infection medium. Moreover, the TEER of the monolayers was measured at each time point. Data are presented as the percentage efficiency of translocation.

\subsection{C. sakazakii cytotoxic effect on the Caco-2 and HBMEC cell lines}

The ability of $C$. sakazakii to induce cytotoxicity was assessed using the colorimetric MTT (3-(4,5-dimethylthiazol-2-yl)-2,5 diphenyltetrazolium bromide) assay. This assay is based on the reduction of MTT (Sigma Aldrich, UK) by viable cells to its insoluble form formazan, which has a purple colour. This experiment was applied as described previously [43,44]. Briefly, bacterial suspensions at a concentration of $4 \times 10^{6} \mathrm{cfu} /$ well (MOI 100) were added to confluent monolayers of the Caco-2 and HBMEC cell lines. The plates were then incubated in $5 \% \mathrm{CO}_{2}$ at $37^{\circ} \mathrm{C}$ for 1 and 3 hours. A volume of 50 $\mu \mathrm{l}$ of MTT was added per $500 \mu \mathrm{l}$ of culture medium. Next, the medium containing MTT was removed and formazan was solubilised in dimethyl sulfoxide (DMSO; Fisher Scientific, UK). The absorbance was measured at $600_{\mathrm{nm}}$ after 3 hours. The negative control for the assay consisted of uninfected cells.

\subsection{C. sakazakii persistence in human macrophages}

As previously given by Townsend et al. [19] with slight modifications, macrophages were grown in RPI medium containing $10 \%(\mathrm{v} / \mathrm{v})$ FCS, $1 \%(\mathrm{v} / \mathrm{v})$ NEAA, and $1 \%(\mathrm{v} / \mathrm{v})$ 
penicillin-streptomycin (Sigma Aldrich, UK), and then treated with growth medium contains $2 \mathrm{mM} \mathrm{L-glutamine,} 10 \mathrm{mM}$ HEPES, and $1 \mathrm{mM}$ sodium pyruvate (Sigma Aldrich, UK) for maturation. Before seeding the 24-well plates, phorbol 12-myristate 13-acetate (PMA; Sigma Aldrich, UK) at a concentration of $0.1 \mu \mathrm{g} / \mathrm{ml}(\mathrm{v} / \mathrm{v})$ was added for promoting cell adhesion. The cells were then plated into 24 -well plates at a concentration of $4 \times 10^{4}$ cell/well and incubated in $5 \% \mathrm{CO}_{2}$ at $37^{\circ} \mathrm{C}$ for 72 hours to get confluent monolayer. Macrophages were infected by overnight-cultured bacterial suspensions with $\mathrm{MOI} 10$. The plates then were incubated 1 hour in $5 \% \mathrm{CO}_{2}$ at $37^{\circ} \mathrm{C}$. After the previous incubation period, the medium was aspirated and replaced with infection medium containing $125 \mu \mathrm{g} / \mathrm{ml}(\mathrm{v} / \mathrm{v})$ of gentamicin and incubated in $5 \% \mathrm{CO}_{2}$ at $37^{\circ} \mathrm{C}$ for 1 hour. Four plates were then washed by PBS and supplied with infection medium contains $50 \mu \mathrm{g} / \mathrm{ml}(\mathrm{v} / \mathrm{v})$ of gentamicin for further incubation. After each time point of incubation, the plates were washed by PBS before lysing by $1 \%(v / v)$ Triton $X-100$, and then serially diluted before plating on TSA to obtain the intracellular bacteria at different time points. Data are displayed in percentage of uptake and persistence.

\subsection{C. sakazakii persistence in human microglial cells}

As previously described by Liu et al. [45] with slight modifications, microglial cells were grown in basal medium containing $10 \%(\mathrm{v} / \mathrm{v}) \mathrm{FCS}, 10 \%(\mathrm{v} / \mathrm{v})$ microglial growth supplement, and $1 \%(\mathrm{v} / \mathrm{v})$ penicillin-streptomycin (Innoprot, Spain) for three days in $75 \mathrm{~cm}^{3}$ tissue culture flask. The cells then were seeded into four 24-well plates at a concentration of $4 \times 10^{4}$ cell/well and incubated in $5 \% \mathrm{CO}_{2}$ at $37^{\circ} \mathrm{C}$ for 48 hours to achieve confluency. Next, the cells were infected by overnight-cultured bacteria with $\mathrm{MOI}$ 10. Afterwards, the plates were incubated for 1 hour in $5 \% \mathrm{CO}_{2}$ at $37^{\circ} \mathrm{C}$. The medium then was aspirated and replaced by infection medium contains $125 \mu \mathrm{g} / \mathrm{ml}$ (v/v) of gentamicin and incubated in $5 \% \mathrm{CO}_{2}$ at $37^{\circ} \mathrm{C}$ for 1 hour after washing by PBS. Three plates were then washed 3 times by PBS and supplied with infection medium contains $50 \mu \mathrm{g} / \mathrm{ml}(\mathrm{v} / \mathrm{v})$ of gentamicin for further incubation. At the end of each time point of incubation, the cells were washed by PBS before lysing with $1 \%$ (v/v) Triton X-100, and plated on TSA after being serially diluted to obtain the intracellular bacteria at different time points. Data are displayed in percentage of uptake and persistence.

\subsection{C. sakazakii sensitivity to human serum}


The sensitivity of $C$. sakazakii strains to active human serum was conducted as described previously by Hughes et al. [46] with slight modification. Bacterial cultures were grown overnight in LB at $37^{\circ} \mathrm{C}$ with shaking at $200 \mathrm{rpm}$ then centrifuged for 10 minutes at $1300 \mathrm{rpm} \_\left(\text {Mikro 200-Hettik). The pellet then was re-suspended to } 10^{6}\right.$ $\mathrm{cfu} / \mathrm{ml}$ in $5 \mathrm{ml}$ of phosphate buffered saline (PBS; Sigma Aldrich, UK). A volume of $0.5 \mathrm{ml}$ of the suspension was added into $1.5 \mathrm{ml}$ of undiluted active human serum (Sigma Aldrich, UK). The samples were loaded into a 24-well plate and incubated at $37^{\circ} \mathrm{C}$. Viable counts were obtained at 4 different time points. Data are displayed in percentage of percent survival of inoculum.

\subsection{PCR probing for cpa gene and BLAST genome search}

Plasmid DNA extraction was carried out according to the manufacturer's instructions using QIAprep Spin Miniprep Kit (Qiagen, UK). PCR primers were designed to target cpa loci on the large C. sakazakii plasmid pESA3 plasmid. GoTaq ${ }^{\circledR}$ DNA Polymerase kit (Promega, UK) was used for all PCR reactions' preparation. Primers design, and the PCRs were all conducted according to Franco et al. [25]. All reactions started with 3 minutes at $94^{\circ} \mathrm{C}$ and denaturation step at $94^{\circ} \mathrm{C}$ for 30 seconds, followed by 25 cycles of $30 \mathrm{~s}$ at $56{ }^{\circ} \mathrm{C}$ and $30 \mathrm{~s}$ at $72{ }^{\circ} \mathrm{C}$. The final extension period was $10 \mathrm{~min}$ at $72{ }^{\circ} \mathrm{C}$. Primer pair cpafw, 5'-GACAACCCTGAGTTCTGGTAAC, and cparv, 5'ATGCGTATTTCTGCTGGTAA, targets a 306 bp region. Moreover, BLAST genome search was applied to strains using the sequence of cpa gene to confirm its presence. The BLAST search was at http://www.pubmlst.org/cronobacter.

\subsection{Statistical analysis}

Data were assessed for normality using Kolmogorov-Smirnov test and normality histograms. The normally distributed data were analysed using the parametric Oneway Analysis of Variance test (ANOVA) with Tukey's post-hoc test, and were expressed as mean values and the standard error of mean (Mean \pm SEM). Data that were not normally distributed were subjected to Kruskal-Wallis test, the nonparametric equivalent of the parametric ANOVA, and were expressed as mean values and the standard deviation (Mean \pm SD). Tukey's post-hoc analysis was performed as a single step multi-comparison test to compare the significance of the means of every $C$. sakazakii strain in relation to other strains as pairwise comparisons. A $P$-value of $<0.05$ was considered statistically significant. Computer
Formatted: Font: (Default) Helvetica, 11 pt, Complex Script Font: $11 \mathrm{pt}$ 
statistical analysis software was used to perform the analysis (IBM SPSS version 22.0, Chicago, IL, USA).

\section{Acknowledgements}

The authors would like to thank King Saud bin Abdulaziz University for health Sciences for the financial support and Nottingham Trent University for the technical support. We also would like to thank Dr. Feda Aljaser for her help in preparing this manuscript.

\section{References}

[1] Tall BD, Chen Y, Yan Q, Gopinath GR, Grim CJ, Jarvis KG, et al. Cronobacter: an emergent pathogen causing meningitis to neonates through their feeds. Science Progress. 2014;97:154-72.

[2] Kucerova E, Clifton SW, Xia XQ, Long F, Porwollik S, Fulton L, et al. Genome sequence of Cronobacter sakazakii BAA-894 and comparative genomic hybridization analysis with other Cronobacter species. PLoS One. 2010;5:e9556.

[3] Baldwin A, Loughlin M, Caubilla-Barron J, Kucerova E, Manning G, Dowson C, et al. Multilocus sequence typing of Cronobacter sakazakii and Cronobacter malonaticus reveals stable clonal structures with clinical significance which do not correlate with biotypes. BMC Microbiology. 2009;9:223-31.

[4] Jackson E, Sonbol H, Masood N, Forsythe S. Genotypic and phenotypic characteristics of Cronobacter species, with particular attention to the newly reclassified species Cronobacter helveticus, Cronobacter pulveris, and Cronobacter zurichensis. Food Microbiology. 2014;44:226-35.

[5] Holy O, Forsythe S. Cronobacter spp. as emerging causes of healthcare-associated infection. Journal of Hospital Infection. 2014;86:169-77.

[6] Joseph S, Cetinkaya E, Drahovska H, Levican A, Figueras MJ, Forsythe SJ. Cronobacter condimenti sp. nov., isolated from spiced meat, and Cronobacter universalis sp. nov., a species designation for Cronobacter sp. genomospecies 1, recovered from a leg infection, water and food ingredients. International Journal of Systematic and Evolutionary Microbiology. 2012;62:1277-83.

[7] Himelright I, Harris E, Lorch V, Anderson M, Jones T, Craig A, et al. Enterobacter sakazakii infections associated with the use of powdered infant formula-Tennessee, 2001. Morbidity and Mortality Weekly Report: CDC; 2002. p. 297-300.

[8] Hariri S, Joseph S, Forsythe SJ. Cronobacter sakazakii ST4 strains and neonatal meningitis, United States. Emerging Infectious Diseases. 2013;19:175.

[9] Baumbach J, Rooney K, Smelser C, Torres P, Bowen A, Nichols M. Cronobacter species isolation in two infants - New Mexico, 2008. Morbidity and Mortality Weekly Report: CDC; 2009. p. $1179-83$.

[10] Bar Oz B, Preminger A, Peleg O, Block C, Arad I. Enterobacter sakazakii infection in the newborn. Acta Paediatrica. 2001;90:356-8.

[11] Bowen $A B$, Braden CR. Invasive Enterobacter sakazakii disease in infants. Emerging Infectious Diseases. 2006;12.

[12] Giri CP, Shima K, Tall BD, Curtis S, Sathyamoorthy V, Hanisch B, et al. Cronobacter spp. (previously Enterobacter sakazakii) invade and translocate across both cultured human intestinal epithelial cells and human brain microvascular endothelial cells. Microbial Pathogenesis. 2011;52:140-7.

[13] Mange JP, Stephan R, Borel N, Wild P, Kim KS, Pospischil A, et al. Adhesive properties of Enterobacter sakazakii to human epithelial and brain microvascular endothelial cells. BMC Microbiology. 2006;6:58. 
[14] Caubilla-Barron J, Hurrell E, Townsend S, Cheetham P, Loc-Carrillo C, Fayet O, et al. Genotypic and phenotypic analysis of Enterobacter sakazakii strains from an outbreak resulting in fatalities in a neonatal intensive care unit in France. Journal of Clinical Microbiology. 2007;45:3979-85.

[15] Forsythe SJ, Dickins B, Jolley KA. Cronobacter, the emergent bacterial pathogen Enterobacter sakazakii comes of age; MLST and whole genome sequence analysis. BMC Genomics 2014;15:1121.

[16] Wilson M, McNab R, Henderson B. Bacterial disease mechanisms: an introduction to cellular microbiology: Cambridge University Press; 2002.

[17] Pagotto FJ, Nazarowec-White M, Bidawid S, Farber JM. Enterobacter sakazakii: infectivity and enterotoxin production in vitro and in vivo. Journal of Food Protection. 2003;66:370-5.

[18] Townsend S, Hurrell E, Forsythe S. Virulence studies of Enterobacter sakazakii isolates associated with a neonatal intensive care unit outbreak. BMC Microbiology. 2008;8:64.

[19] Townsend S, Hurrell E, Gonzalez-Gomez I, Lowe J, Frye JG, Forsythe S, et al. Enterobacter sakazakii invades brain capillary endothelial cells, persists in human macrophages influencing cytokine secretion and induces severe brain pathology in the neonatal rat. Microbiology. 2007;153:3538-47.

[20] Townsend S, Caubilla Barron J, Loc-Carrillo C, Forsythe S. The presence of endotoxin in powdered infant formula milk and the influence of endotoxin and Enterobacter sakazakii on bacterial translocation in the infant rat. Food Microbiology. 2007;24:67-74.

[21] Franco A, Kothary M, Gopinath G, Jarvis K, Grim C, Hu L, et al. Cpa, the outer membrane protease of Cronobacter sakazakii, activates plasminogen and mediates resistance to serum bactericidal activity. Infection and Immunity. 2011;79:1578-87. [22] Joseph S, Desai P, Ji Y, Cummings CA, Shih R, Degoricija L, et al. Comparative analysis of genome sequences covering the seven Cronobacter species. PLoS One. 2012;7:e49455. [23] Kim K, Kim KP, Choi J, Lim JA, Lee J, Hwang S, et al. Outer membrane proteins A $(\mathrm{OmpA})$ and $\mathrm{X}(\mathrm{OmpX})$ are essential for basolateral invasion of Cronobacter sakazakii. Applied and Environmental Microbiology. 2010;76:5188-98.

[24] Singamsetty VK, Wang Y, Shimada H, Prasadarao NV. Outer membrane protein A expression in Enterobacter sakazakii is required to induce microtubule condensation in human brain microvascular endothelial cells for invasion. Microbial Pathogenesis. 2008;45:181-91.

[25] Franco A, Hu L, Grim C, Gopinath G, Sathyamoorthy V, Jarvis K, et al. Characterization of putative virulence genes on the related RepFIB plasmids harbored by Cronobacter spp. Applied and Environmental Microbiology. 2011;77:3255-67.

[26] Alzahrani H, Winter J, Boocock D, De Girolamo L, Forsythe SJ. Characterisation of outer membrane vesicles from a neonatal meningitic strain of Cronobacter sakazakii. FEMS Microbiology Letters. 2015:fnv085.

[27] Polazzi E, Monti B. Microglia and neuroprotection: from in vitro studies to therapeutic applications. Progress in Neurobiology. 2010;92:293-315.

[28] Guidi-Rontani C. The alveolar macrophage: the Trojan horse of Bacillus anthracis. Trends in Microbiology. 2002;10:405-9.

[29] Baggiolini M, Loetscher P, Moser B. Interleukin-8 and the chemokine family. International Journal of Immunopharmacology. 1995;17:103-8.

[30] Baggiolini M, Dewald B, Moser B. Interleukin-8 and related chemotactic cytokines-CXC and CC chemokines. Advances in Immunology. 1993;55:97-179.

[31] Sprenger H, Rösler A, Tonn P, Braune H, Huffmann G, Gemsa D. Chemokines in the cerebrospinal fluid of patients with meningitis. Clinical Immunology and Immunopathology. 1996;80:155-61.

[32] Koedel U, Bayerlein I, Paul R, Sporer B, Pfister H. Pharmacologic interference with NF$\mathrm{KB}$ activation attenuates central nervous system complications in experimental pneumococcal meningitis. Journal of Infectious Diseases. 2000;182:1437-45.

[33] Hanke ML, Kielian T. Toll-like receptors in health and disease in the brain: mechanisms and therapeutic potential. Clinical Science. 2011;121:367-87.

[34] Taylor PW. Bactericidal and bacteriolytic activity of serum against gram-negative bacteria. Microbiological Reviews. 1983;47:46.

[35] Rautemaa R, Meri S. Complement-resistance mechanisms of bacteria. Microbes and Infection. 1999;1:785-94. 
889 [36] Schwizer S, Tasara T, Zurfluh K, Stephan R, Lehner A. Identification of genes involved in 890 serum tolerance in the clinical strain Cronobacter sakazakii ES5. BMC Microbiology. $8912013 ; 13: 38$.

892 [37] Joseph S, Forsythe SJ. Predominance of Cronobacter sakazakii sequence type 4 in 893 neonatal infections. Emerging Infectious Diseases. 2011;17:1713-5.

894 [38] Burns JL, Griffith A, Barry JJ, Jonas M, Chi EY. Transcytosis of gastrointestinal epithelial 895 cells by Escherichia coli K1. Pediatric research. 2001;49:30-7.

896 [39] Finlay BB, Falkow S. Salmonella interactions with polarized human intestinal Caco-2 897 epithelial cells. Journal of Infectious Diseases. 1990;162:1096-106.

898 [40] Nizet V, Kim K, Stins M, Jonas M, Chi EY, Nguyen D, et al. Invasion of brain

899 microvascular endothelial cells by group B streptococci. Infection and Immunity. 900 1997;65:5074-81.

901 [41] Badger JL, Stins MF, Kim KS. Citrobacter freundii invades and replicates in human brain 902 microvascular endothelial cells. Infection and Immunity. 1999;67:4208-15.

903 [42] Kim KS. Pathogenesis of bacterial meningitis: from bacteraemia to neuronal injury. 904 Nature Reviews Neuroscience. 2003;4:376-85.

905 [43] Krzymińska S, Mokracka J, Koczura R, Kaznowski A. Cytotoxic activity of Enterobacter 906 cloacae human isolates. FEMS Immunology \& Medical Microbiology. 2009;56:248-52.

907 [44] Kielian T, McMahon M, Bearden ED, Baldwin AC, Drew PD, Esen N. S 908 aureus dependent microglial activation is selectively attenuated by the cyclopentenone 909 prostaglandin 15 deoxy $\Delta 12,14$ prostaglandin J2 (15d PGJ2). Journal of Neurochemistry. 910 2004;90:1163-72.

911 [45] Liu S, Kielian T. Microglial activation by Citrobacter koseri is mediated by TLR4-and 912 MyD88-dependent pathways. The Journal of Immunology. 2009;183:5537-47.

913 [46] Hughes C, Phillips R, Roberts A. Serum resistance among Escherichia coli strains 914 causing urinary tract infection in relation to $\mathrm{O}$ type and the carriage of hemolysin, colicin, and 915 antibiotic resistance determinants. Infection and Immunity. 1982;35:270-5. 